\title{
summary
}

\section{Results of guided tissue regeneration are highly variable}

Needleman IG, Giedrys-Leeper E, Tucker RJ, Worthington HV. Guided tissue regeneration for periodontal infra-bony defects (Cochrane Review). In: The Cochrane Library, Issue 2, 2001. Oxford: Update Software

Objective To assess the efficacy of guided tissue regeneration (GTR) in the treatment of periodontal infra-bony defects measured against the current standard of surgical periodontal treatment, openflap debridement.

Data sources The Cochrane Oral Health Group specialised trials register, Medline, hand searches of the Journal of Periodontology, Clinical Periodontology, Periodontal Research and bibliographies of all relevant papers and review articles, up to October 2000. In addition, experts, groups and companies involved in surgical research were contacted to find other trials or unpublished material or to clarify ambiguous or missing data. Requests for data were also posted on two periodontal electronic discussion groups.

Study selection Randomised controlled trials (RCT) of at least 12 months' duration comparing GTR (with or without graft materials) with open-flap debridement for the treatment of periodontal infrabony defects. Furcation involvement and studies specifically treating early onset diseases were excluded.

Data extraction and synthesis Initial screening of studies was conducted independently by two reviewers and data abstraction by three reviewers, with methodological quality assessed using both individual components and the Jadad scale. Inter-rater agreement was determined by Kappa scores. Methodological quality was used in sensitivity analyses to test the robustness of the conclusions. The results were expressed as weighted mean differences (WMD) and 95\% confidence intervals (CI) for continuous outcomes and as relative risk (RR) and 95\% CI for dichotomous outcomes, calculated using random-effects models where significant heterogeneity was detected $(P<0.1)$. The primary outcome measure was gain in clinical attachment.

Results Eleven studies were finally included in the review, 10 testing GTR alone and two testing GTR plus bone substitutes (one study had both test treatment arms). Statistically significant differences were found for attachment level change, probing depth reduction and hard tissue probing at surgical re-entry (see Table 1). Heterogeneity between studies was highly statistically significant for all principal comparisons and could not be explained satisfactorily by sensitivity analyses. The quality of study-reporting was low, with seven out of 11 studies graded as poor using the Jadad score. The number needed to treat (NNT) for GTR to achieve one extra site gaining $2 \mathrm{~mm}$ or more attachment over
Table 1 Summary of meta-analysis

\begin{tabular}{|c|c|c|c|}
\hline & \multicolumn{3}{|c|}{$\begin{array}{l}\text { Weighted mean difference } \\
(\mathrm{mm})(95 \% \mathrm{Cl})\end{array}$} \\
\hline & $\begin{array}{l}\text { Attachment } \\
\text { level } \\
\text { change }\end{array}$ & $\begin{array}{l}\text { Probing } \\
\text { depth } \\
\text { reduction }\end{array}$ & $\begin{array}{c}\text { Hard tissue } \\
\text { probing at } \\
\text { surgical } \\
\text { re-entry }\end{array}$ \\
\hline $\begin{array}{l}\text { GTR alone and } \\
\text { open-flap debridement }\end{array}$ & $\begin{array}{c}1.11 \\
(0.63-1.59)\end{array}$ & $\begin{array}{c}0.80 \\
(0.14-1.46)\end{array}$ & $\begin{array}{c}1.39 \\
(1.08-1.71)\end{array}$ \\
\hline $\begin{array}{l}\text { GTR plus bone substitutes } \\
\text { and open-flap debridement }\end{array}$ & $\begin{array}{c}1.25 \\
(0.89-1.61) \\
\end{array}$ & $\begin{array}{c}1.24 \\
(0.89-1.59) \\
\end{array}$ & $\begin{array}{c}3.37 \\
(3.14-3.61) \\
\end{array}$ \\
\hline
\end{tabular}

open-flap debridement was eight (95\% CI, 4-33), based on an incidence of $32 \%$ of sites in the control group failing to gain $2 \mathrm{~mm}$ or more of attachment. For baseline incidences in the range of the control groups of $10 \%$ and $55 \%$ the NNTs are 24 and 3.

Conclusions Overall, GTR was a little more effective in increasing attachment gain, reducing pocket depth and favouring gain in hard tissue probing at re-entry surgery. The marked variability between studies limits conclusions about the clinical benefit of GTR, however. There is evidence that GTR can produce a significant improvement over conventional open-flap surgery but the factors responsible for success or failure are not clear from the literature. We recommend that future trials should follow the CONSORT guidelines both in their conduct and reporting. Studies should aim to identify factors associated with achieving consistent benefits over open-flap surgery and these benefits should include traditional probing outcomes as well as considering patient-centred endpoints. Open-flap surgery should remain the control comparison in these studies

Evidence-Based Dentistry (2002) 3, 12-13. DOI: 10.1038/sj/ ebd/6400083

Address for reprints: Dr lan Needleman, Lecturer, Department of Periodontology, Eastman Dental Institute, 256 Gray's Inn Road, London WC1X 8LD, UK. E-mail: i.needleman@eastman.ucl.ac.uk

\section{Commentary}

Chronic periodontitis is the most common form of periodontal disease, affecting up to $30 \%$ of the adult population. ${ }^{1}$ The ultimate goal of periodontal therapy has been regeneration of tissues lost due to disease, but this goal remains elusive. Traditional surgical therapy involves flap surgery, debridement of the affected area and, often, osseous recontouring. Although periodontal pocket depths are decreased, the results may be less than ideal from the patient's point of view, as there may be an increase in dentinal hypersensitivity and a negative change in aesthetics.
During GTR therapy a partially occlusive barrier membrane is placed between the healing flap and the root/ bone surface. The principle behind the use of this membrane is to exclude the rapidly proliferating epithelial cells from migrating down the healing wound surface. Theoretically, this allows the slower 
moving periodontal ligament cells and osteogenic cells to migrate into the wound, regenerating lost tissue. In addition to unknown efficacy, the drawbacks of GTR therapy are increased expense and, in the case of a nonresorbable membrane, a second surgical procedure.

This systematic review synthesises the literature on the use of GTR in the treatment of infra-bony defects (ie, bony craters). Much has been written over the last 25 years in this area. Needleman et al. found 11 randomised controlled trials that met their review's inclusion criteria. Unfortunately, the quality of most of these studies was poor: many publications presented incomplete methods or results, and outcomes measured were highly variable between studies. Of the 10 studies dealing with GTR alone (ie, no bone substitutes) versus traditional flap surgery, seven received a quality score of less than 3 (out of a possible 4) - poor quality reports. Of the remaining four studies, one scored 3, and the others, 4 . It bears repeating that all future clinical researchers (not just those in periodontal regenerative research) should follow the CONSORT guidelines in both the conduct of trials and the reporting of results.

Meta-analysis revealed that the weighted mean difference in probing depth and attachment level between GTR and traditional surgery was 0.80 and $1.11 \mathrm{~mm}$, respectively. If we presume that the average changes in probing depth and attachment level were calculated using just the sites treated and not the entire mouth or sextant, and accounting for potential measurement error of 1-2 mm with a traditional periodontal probe, these differences do not appear to be clinically significant. The addition of bone substitutes did not significantly alter the results. Although the difference in hard tissue probing is higher, it does not seem to impact on attachment level changes.

A more meaningful outcome than pocket depth or attachment level change would be the number of sites gaining at least $2 \mathrm{~mm}$ of attachment. This threshold is one that is more likely to alter management decisions on the part of the clinician. From the analysis in this review it was determined that eight sites would need to be treated with GTR for one site to show $2 \mathrm{~mm}$ attachment gain (ie, NNT). It is rare that only one site per tooth is affected by an infra-bony defect. Yet even if an average of four sites per tooth were affected, and two teeth were treated with GTR in a particular patient, results from this review suggest only one site on those two teeth would show significant clinical improvement. For those who are risk-averse, these do not seem like favourable odds. For others, it might be worth the gamble (and the additional costs), especially in areas of aesthetic concern.

This review found the results between studies or centres were highly variable. In the 10 studies examining GTR alone, the changes in clinical attachment ranged from 0.02$2.60 \mathrm{~mm}$. Analysis performed with and without results of the poorer quality studies could not explain the heterogeneity. There are many possible explanations for this. Part of the difference may be due to differences in accounting for confounders (or prognostic factors) such as plaque levels, cigarette smoking and baseline severity of the defect. Another is that this is a technically sensitive procedure and thus highly surgeon-dependent. Noting that two of the studies showed twice the attachment-level gain of the overall group estimate, this hypothesis seems to have some merit.

As with many clinical trials in dentistry, none of these investigations reported patient-based outcomes. The likelihood of post-operative complications, changes in aesthetics or future tooth loss can play a significant role in how patients choose between alternative therapies. Thus, in addition to identifying factors associated with successful outcomes of GTR therapy, some consideration should be given to measuring quality-of-life outcomes in future trials.

From the details of this rigorous, welldocumented review it is clear that any positive reported results must be viewed with caution. It appears that a method that can consistently regenerate periodontal tissue lost because of disease eludes us still.

1. Oliver $\mathrm{R}$, Brown $\mathrm{L}$, Loe $\mathrm{H}$. Variations in the prevalence and extent of periodontitis. J Am Dent Assoc 1991; 122:43-48.

\section{Debora C Matthews Division of Periodontics, Faculty of Dentistry, Dalhousie University, Halifax, Nova Scotia, Canada}

